\title{
The association between longer relative leukocyte telomere length and risk of glioma is independent of the potentially confounding factors allergy, BMI, and smoking
}

\author{
Ulrika Andersson $^{1}$ (D) Sofie Degerman ${ }^{2} \cdot$ Anna M. Dahlin $^{1} \cdot$ Carl Wibom $^{1} \cdot$ Gunnar Johansson $^{1} \cdot$ Melissa L. Bondy $^{3}$. \\ Beatrice S. Melin ${ }^{1}$
}

Received: 3 July 2018 / Accepted: 6 December 2018 / Published online: 17 December 2018

(c) The Author(s) 2018

\begin{abstract}
Purpose Previous studies have suggested an association between relative leukocyte telomere length (rLTL) and glioma risk. This association may be influenced by several factors, including allergies, BMI, and smoking. Previous studies have shown that individuals with asthma and allergy have shortened relative telomere length, and decreased risk of glioma. Though, the details and the interplay between rLTL, asthma and allergies, and glioma molecular phenotype is largely unknown.

Methods rLTL was measured by qPCR in a Swedish population-based glioma case-control cohort (421 cases and 671 controls). rLTL was related to glioma risk and health parameters associated with asthma and allergy, as well as molecular events in glioma including IDH1 mutation, 1p/19q co-deletion, and EGFR amplification.

Results Longer rLTL was associated with increased risk of glioma (OR=1.16; 95\% CI 1.02-1.31). Similar to previous reports, there was an inverse association between allergy and glioma risk. Specific, allergy symptoms including watery eyes was most strongly associated with glioma risk. High body mass index (BMI) a year prior diagnosis was significantly protective against glioma in our population. Adjusting for allergy, asthma, BMI, and smoking did not markedly change the association between longer rLTL and glioma risk. rLTL among cases was not associated with IDH1 mutation, 1p/19q codeletion, or EGFR amplification, after adjusting for age at diagnosis and sex.

Conclusions In this Swedish glioma case-control cohort, we identified that long rLTL increases the risk of glioma, an association not confounded by allergy, BMI, or smoking. This highlights the complex interplay of the immune system, rLTL and cancer risk.
\end{abstract}

Keywords Glioma $\cdot$ Relative leukocyte telomere length (rLTL) $\cdot$ Allergy $\cdot$ BMI $\cdot$ Smoking $\cdot$ IDH1 $\cdot 1$ p/19q $\cdot$ EGFR

\section{Introduction}

Glioma, a malignant brain tumor confers a poor prognosis, and there are few established etiological factors. These include, rare inherited syndromes such as neurofibromatosis type I and II, LiFraumeni syndrome [1], and high dose ionizing radiation have been associated with increased risk of glioma development [2, 3]. Asthma and respiratory allergies have consistently been reported to be protective against glioma [4-7]. A number of common genetic variants have also been associated with glioma risk [8-10]. Both studies of familial and sporadic glioma have shown associations between glioma risk and genetic variants mapping to genes functionally important in telomere regulation, e.g., RTEL, TERT, TERC [8, 11], POT1 [12], and OBFC1 [13]. 
Telomeres, which are located at the chromosome ends, have important functions in sustaining cellular chromosomal stability. Since telomeres are shortened during replication, shorter telomere length is observed in aging tissues. Individuals with asthma in adulthood have shorter leukocyte telomeres compared to healthy controls [14]. Additionally, increased BMI and cigarette smoking are also known to be associated with shorter telomere length in white blood cells [15]. Short telomere length measured in surrogate tissues such as peripheral blood has been associated with increased risk of many diseases, including cardiovascular and respiratory diseases, as well as cancer [16]. Telomere length heterogeneity appears to be present in brain tumors, since a certain subtype of glioblastoma seems to up-regulate telomerase through highly clonal TERT promoter mutations, whereas other subtypes of glioblastoma maintain telomere length through subclonal mutations activating an alternative lengthening of telomeres pathway (ALT) [17]. Evidence suggests that there may be a link between glioma risk and telomere length, including the commonality that both telomere length and glioma have been associated with allergy and asthma.

IDH1 mutations and co-deletion of the $1 \mathrm{p}$ and $19 \mathrm{q}$ chromosomal arms (1p/19q co-deletion) are two of the main genetic biomarkers important for glioma classification and diagnosis [18]. A previously published study has shown that glioma with $I D H 1$ mutations and lack of $1 \mathrm{p} / 19 \mathrm{q}$ codeletion are negative for both hTERT expression and telomerase activity, and cases have significantly longer tumor tissue telomeres [19]. EGFR is frequently overexpressed in primary glioblastoma versus secondary glioblastoma and is a characteristic of a more aggressive phenotype. It has been shown that EGFR overexpression leads to phosphorylation of hTERT through the PI3K/AKT signaling pathway, resulting in the activation of telomerase [20]. Nevertheless, any potential link between rLTL in peripheral blood and glioma risk is not well characterized. Genotypically-estimated leukocyte telomere length is reported to be associated with glioma risk [21], but only a small number of studies and with contradictory results have measured rLTL in peripheral blood in glioma cases and controls [22, 23], reviewed in [24].

In this study, we investigated a population-based case-control series from Sweden with a quantitative-PCR method for rLTL, examining with direct measurement of the potential association between rLTL and risk of glioma. We controlled for age at diagnosis/enrollment, sex, allergy, asthma, smoking history, and BMI, as these are established factors associated with telomere length and, to some extent, glioma. Furthermore, we examined the association between rLTL, and molecular events in gliomagenesis, including $I D H 1$ mutation, $1 \mathrm{p} / 19 \mathrm{q}$ co-deletion, and $E G F R$ amplification.

\section{Study subjects and methods}

\section{Ascertainment and collection of study subjects}

We included all Swedish participants from two international multi-center efforts aimed at exploring glioma etiology: the Genetic Epidemiology of Glioma International Consortium (GLIOGENE) study [25], and the Glioma International Case-Control (GICC) study [26]. The GLIOGENE study included cases with hereditary disease diagnosed between 2007 and 2013 [25]. Cases with a reported or confirmed diagnosis of neurofibromatosis I, neurofibromatosis II, Turcot's syndrome, or tuberous sclerosis were excluded from the GLIOGENE study. The GICC study included sporadic cases diagnosed between 2010 and 2013, as well as population-based controls (aged 18-80, frequency matched to cases based on age at diagnosis and sex). The methods for each of these studies have been previously described $[25,26]$. Study participants of GLIOGENE and GICC contributed biological samples and completed questionnaires. The GICC questionnaire included questions related to allergies, asthma, smoking history, and BMI. All sites received Institutional Review Board or ethical board approval to conduct the study, and informed consent was obtained from participants. Ultimately, we included 37 Swedish GLIOGENE-cases (36 probands and one affected relative) and 384 Swedish GICC-cases, as well as 671 population-based GICC-controls, and characteristics of the study subjects are shown in Table 1. For the analyses of certain subgroups of glioma, we used one or two age- and sex- matched controls for each case, since these glioma subgroups are known to be correlated with both age and sex (Table 2). From the questionnaires used for the GICC study, we extracted data on allergy status (experienced symptoms: skin/respiratory/watery eyes) and asthma (diagnosed by doctor) as well as two smoking variables: current smoking (yes/no) and ever smoking (yes/no), defined as "> 100 cigarettes in a lifetime" [26] (Table 1). We also extracted BMI, which was categorized as underweight/normal $(\mathrm{BMI}<25)$ and overweight/obese $(B M I \geq 25)$ (Table 1). In collaboration with the Swedish GICC study centers, we obtained unstained slides from paraffin embedded tissue for 367 of the glioma patients. These slides were used in the immunohistochemistry (IHC) and fluorescent in situ hybridization (FISH) analyses (described below).

\section{Relative telomere length (rLTL) PCR}

Genomic DNA was extracted from the buffy coat from EDTA-venous blood samples using FlexiGene DNA Kit 
Table 1 Characteristics of cases and matched controls identified through the GLIOGENE- and GICC-studies, and included in the relative telomere length analyses

\begin{tabular}{|c|c|c|c|c|}
\hline & Controls $n(\%)$ & $\begin{array}{l}\text { Cases all glioma } \\
\text { cases } n(\%)\end{array}$ & $\begin{array}{l}\text { Low-grade glioma } \\
\text { cases }^{\mathrm{a}} n(\%)\end{array}$ & $\begin{array}{l}\text { High-grade } \\
\text { glioma cases } \\
n(\%)\end{array}$ \\
\hline \multicolumn{5}{|l|}{ Sex } \\
\hline Male & $400(59.6)$ & $250(59.4)$ & $87(51.5)$ & $162(64.8)$ \\
\hline Female & $271(40.4)$ & $171(40.6)$ & $82(48.5)$ & $88(35.2)$ \\
\hline \multicolumn{5}{|c|}{ Diagnosis/enrollment years } \\
\hline $18-29$ years & $6(0.9)$ & $21(5.0)$ & $18(10.6)$ & $3(1.2)$ \\
\hline $30-39$ years & $34(5.0)$ & $34(8.0)$ & $24(14.2)$ & $10(4.0)$ \\
\hline $40-49$ years & $79(11.8)$ & $59(14.0)$ & $31(18.3)$ & $28(11.2)$ \\
\hline $50-59$ years & $198(29.5)$ & $109(25.9)$ & $41(24.3)$ & $68(27.2)$ \\
\hline $60-69$ years & $248(37.0)$ & $148(35.2)$ & $39(23.1)$ & $107(42.8)$ \\
\hline $70-80$ years & $106(15.8)$ & $50(11.9)$ & $16(9.5)$ & $34(13.6)$ \\
\hline \multicolumn{5}{|c|}{ Allergies (skin, respiratory, watery eyes, digestive problems, anaphylaxis, or other) } \\
\hline Yes & $190(28.3)$ & $82(19.5)$ & $36(21.3)$ & $45(18.0)$ \\
\hline No & $428(63.8)$ & $281(66.7)$ & $102(60.4)$ & $178(71.2)$ \\
\hline Missing & $53(7.9)$ & $58(13.8)$ & $31(18.3)$ & $27(10.8)$ \\
\hline \multicolumn{5}{|c|}{ Asthma (diagnosed by doctor) } \\
\hline Yes & $64(9.5)$ & $35(8.3)$ & $15(8.9)$ & $19(7.6)$ \\
\hline No & $599(89.3)$ & $348(82.7)$ & $136(80.5)$ & $211(84.4)$ \\
\hline Missing & $8(1.2)$ & $38(9.0)$ & $18(10.6)$ & $20(8.0)$ \\
\hline \multicolumn{5}{|c|}{ Ever smoking (smoked more than 100 cigarettes in life time) } \\
\hline Yes & $363(54.1)$ & $207(49.2)$ & $76(42.6)$ & $129(51.6)$ \\
\hline No & $299(44.6)$ & $168(39.9)$ & $72(45.0)$ & $96(38.4)$ \\
\hline Missing & $9(1.3)$ & $46(10.9)$ & $21(12.4)$ & $25(10.0)$ \\
\hline \multicolumn{5}{|c|}{ Current smoking (currently smoke cigarettes) } \\
\hline Yes & $70(10.4)$ & $32(7.6)$ & $13(7.7)$ & $19(7.6)$ \\
\hline No & $293(43.7)$ & $175(41.6)$ & $63(37.3)$ & $110(44.0)$ \\
\hline Missing & $308(45.9)$ & $214(50.8)$ & $93(55.0)$ & $121(48.4)$ \\
\hline \multicolumn{5}{|c|}{ BMI at age 18 (calculated as weight in kilograms divided by height in meters squared) } \\
\hline Valid & $551(82.1)$ & $294(69.8)$ & $119(70.4)$ & $173(69.2)$ \\
\hline Missing & $120(17.9)$ & $127(30.2)$ & $50(29.6)$ & $77(30.8)$ \\
\hline \multicolumn{5}{|c|}{ BMI as adult (calculated as weight in kilograms divided by height in meters squared) } \\
\hline Valid & $630(93.9)$ & $350(83.1)$ & $142(84.0)$ & $206(82.4)$ \\
\hline Missing & $41(6.1)$ & $71(16.9)$ & $27(16.0)$ & $44(17.6)$ \\
\hline \multicolumn{5}{|c|}{ BMI a year prior diagnosis (calculated as weight in kilograms divided by height in meters squared) } \\
\hline Valid & $668(99.6)$ & $367(87.2)$ & $145(85.8)$ & $220(88.0)$ \\
\hline Missing & $3(0.4)$ & $54(12.8)$ & $24(14.2)$ & $30(12.0)$ \\
\hline
\end{tabular}

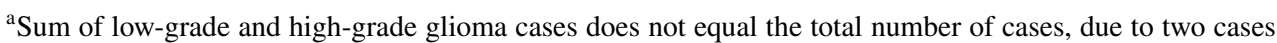
with unclassified tumors
(Qiagen) and DNA purity and concentration were measured by the Nanodrop instrument (Thermo Fisher Scientific). rLTL was determined by the quantitative-PCR method described by Cawthon [27]. Briefly, Telomere (Tel) and single copy gene (HBG) reactions were run separately in triplicates. Tel/HBG (T/S) values were calculated by $2^{-\Delta \mathrm{Ct}}$ method, where $\Delta \mathrm{Ct}=$ average $\mathrm{Ct}_{\mathrm{Tel}}-$ average $\mathrm{Ct}_{\mathrm{HBG}}$. rLTL was generated by dividing a samples $\mathrm{T} / \mathrm{S}$ value with the T/S value of a reference cell line DNA (CCRF) included in all runs. A standard curve generated by the reference DNA was also included in each run to monitor PCR efficiency. The mean inter-assay coefficient of variation for rLTL in this study was $7 \%$.

\section{Immunohistochemistry (IHC)}

Based on eosin staining, a neuropathologist reviewed all slides to ensure that there was adequate tumor tissue in each slide. Slides were pre-treated and stained against IDH1-R132H as previously described [28], using Ultra view DAB kit 760 (Roche, Basel Switzerland) and mouse anti-IDH1-R132H (Dianova, Hamburg, Germany). Slides 
Table 2 Logistic regression models to investigate the association between rLTL and risk of glioma subgroups based on IDH1 mutation, $1 \mathrm{p} / 19 \mathrm{q}$ co-deletion, and EGFR amplification. Glioma grade II-III are defined as non-GBM, and glioma grade IV are defined as GBM

\begin{tabular}{|c|c|c|c|c|}
\hline & Cases $^{\mathrm{a}} n$ & Controls $^{\mathrm{b}} n$ & OR $(95 \% \mathrm{CI})^{\mathrm{c}}$ & $P$ value \\
\hline rLTL in all glioma & 321 & 598 & $1.15(1.00-1.32)$ & 0.046 \\
\hline rLTL in glioma with $I D H I$ wildtype & 265 & 501 & $1.16(0.99-1.35)$ & 0.067 \\
\hline rLTL in glioma with $I D H 1$ mutation and without $1 \mathrm{p} / 19 \mathrm{q}$ co-deletion & 25 & 40 & $1.48(0.86-2.55)$ & 0.155 \\
\hline rLTL in glioma with $\mathrm{I} D H 1$ mutation and $1 \mathrm{p} / 19 \mathrm{q}$ co-deletion & 23 & 43 & $0.92(0.55-1.56)$ & 0.768 \\
\hline rLTL in glioma with $E G F R$ amplification & 100 & 192 & $1.11(0.86-1.44)$ & 0.423 \\
\hline rLTL in all non-GBM & 122 & 211 & $1.13(0.91-1.41)$ & 0.259 \\
\hline rLTL in non-GBM with $I D H 1$ wildtype & 75 & 131 & $1.28(0.95-1.73)$ & 0.108 \\
\hline rLTL in non-GBM with $I D H 1$ mutation and without $1 \mathrm{p} / 19 \mathrm{q}$ co-deletion & 20 & 30 & $1.31(0.70-2.46)$ & 0.400 \\
\hline rLTL in non-GBM with $I D H 1$ mutation and $1 \mathrm{p} / 19 \mathrm{q}$ co-deletion & 21 & 39 & $0.82(0.48-1.42)$ & 0.484 \\
\hline rLTL in non-GBM with $E G F R$ amplification & 13 & 25 & $1.18(0.56-2.47)$ & 0.659 \\
\hline rLTL in all GBM & 199 & 387 & $1.16(0.97-1.39)$ & 0.097 \\
\hline rLTL in GBM with $I D H 1$ wildtype & 190 & 370 & $1.11(0.92-1.34)$ & 0.256 \\
\hline rLTL in GBM with $I D H 1$ mutation & 8 & 15 & $3.65(1.14-11.72)$ & 0.029 \\
\hline rLTL in GBM with $E G F R$ amplification & 87 & 167 & $1.11(0.84-1.47)$ & 0.485 \\
\hline
\end{tabular}

were de-identified to remove any bias, and analyzed by a neuropathologist.

\section{Fluorescent in situ hybridization (FISH)}

Slides were deparaffinized in xylene and dehydrated in absolute alcohol. Slides were placed in a pre-treatment solution for $12 \mathrm{~min}$ at $80{ }^{\circ} \mathrm{C}$ and protease solution for $45 \mathrm{~min}$ at $37^{\circ} \mathrm{C}$ (according to the instruction in Vysis pretreatment kit IV (Abbot, Abbott Park, Illinois). Slides were washed in $\mathrm{H}_{2} \mathrm{O}$ and dehydrated in a series of $70 \%, 80 \%$ and absolute ethanol. Each section was covered with Vysis LSI 1p36 / LSI 1q25 and LSI 19q13/19p13 dual-color probe (Abbott) for detection of chromosome $1 \mathrm{p}$ or $19 \mathrm{q}$ and Vysis $E G F R / C E P 7$ FISH probe Kit (Abbott) for detection $E G F R$ amplification. The sections were co-denatured at $80{ }^{\circ} \mathrm{C}$ for $6 \mathrm{~min}$ and hybridized over night at $37^{\circ} \mathrm{C}$. Unspecific staining was removed using wash buffer one and two according to the instruction in the Vysis pre-treatment kit IV (Abbott). Sections were counterstained using DAPIAntifade (Cytocell, Cambridge, UK). Slides were coded to remove any bias, and two independent observers analyzed each slide. Tumors with deletion in more than $12 \%$ of the cells for chromosome $1 \mathrm{p}$ and $20 \%$ of the cells for chromosome $19 q$ were defined as co-deleted. A ratio between the locus specific EGFR probe and the control probe $C E P 7$ ( $E G F R / C E P 7)$ was calculated were ratios equal to 1 was considered as normal, while more than $10 \%$ cells with a ratio between one and two was considered as chromosomal gain, and more than $10 \%$ cells with a ratio greater than two was considered as amplification.

\section{Statistical analyses}

Odds ratios (ORs), 95\% confidence intervals (CIs), and $P$ values for glioma risk were calculated using unconditional logistic regression models adjusted for age at diagnosis (cases)/enrollment (controls) and sex using rLTL as a continuous variable. The continuous rLTL variable was scaled to unit variance by dividing by its standard deviation, prior to statistical modeling to facilitate model interpretation. Linear regression analyses were conducted to examine the correlation between rLTL, IDH1 mutation, 1p/19q co-deletion, and EGFR amplification in glioma cases. All statistical analyses were performed using the SPSS Statistics 24.0 software (IBM), and $P<0.05$ was considered statistically significant.

\section{Results}

\section{Telomere length and glioma risk}

Longer rLTL in peripheral blood was associated with increased risk of glioma $(\mathrm{OR}=1.16$; 95\% CI 1.02-1.31, $P=0.025)$ (Fig. 1), and this association rely mainly among females $(\mathrm{OR}=1.29 ; 95 \% \mathrm{CI} 1.40-13.91, P=0.011)$ compared to males $(\mathrm{OR}=1.07 ; 95 \% \mathrm{CI} 0.57-3.76, P=0.429)$ (data not shown). Although not statistically significant, the association was in the same direction in all investigated glioma subgroups, with the exception of glioma with IDH1 mutation and $1 \mathrm{p} / 19 \mathrm{q}$ co-deletion (for all glioma with IDH 1 mutation and $1 \mathrm{p} / 19 \mathrm{q}$ co-deletion $\mathrm{OR}=0.92 ; 95 \% \mathrm{CI}$ $0.55-1.56$, and for non-GBM (glioma grade II-III) with 
A)

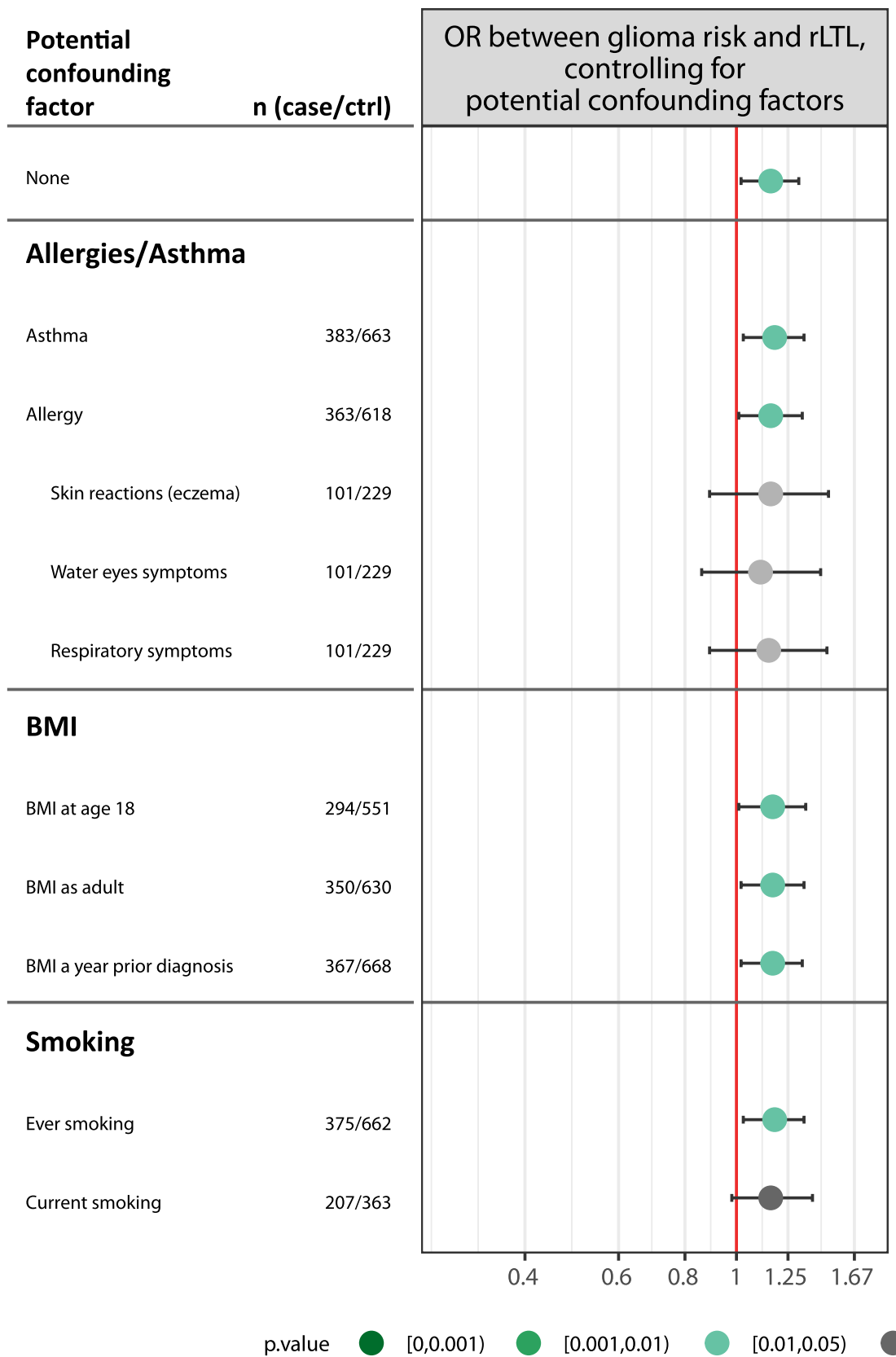

B)

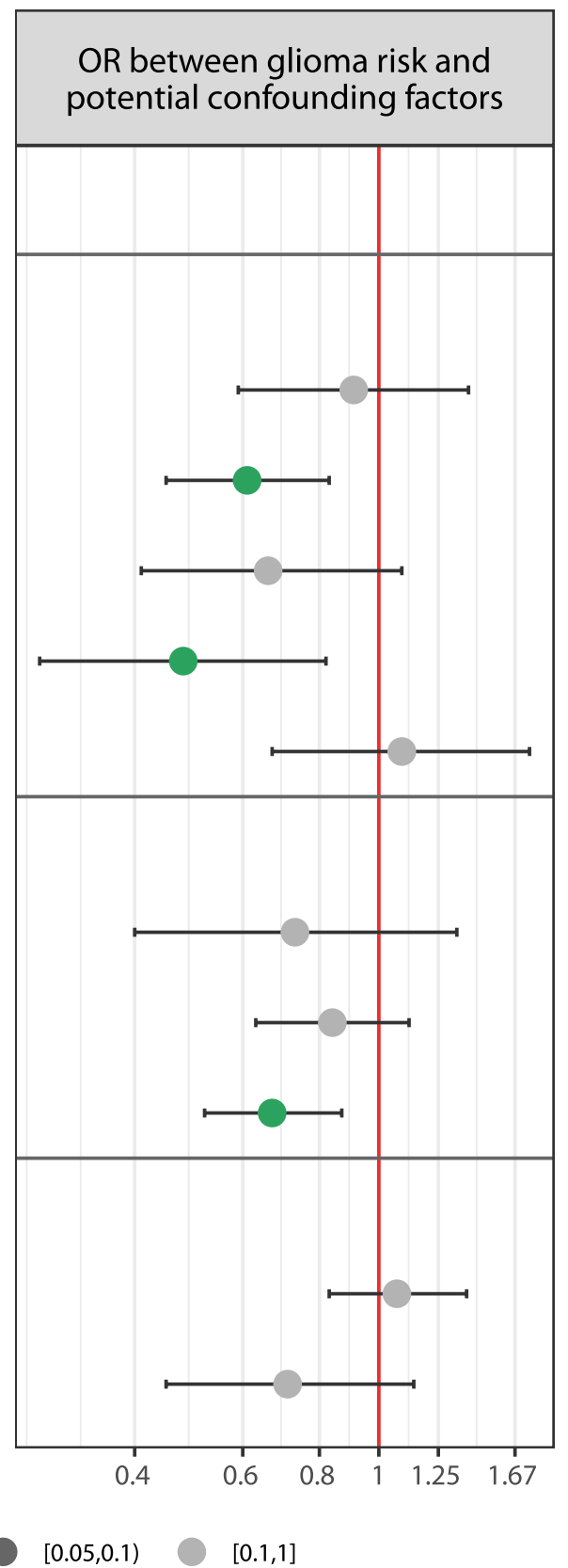

Fig. 1 a Forest plot illustrating the association between glioma risk and rLTL, controlling for the potential confounding factors; asthma (no asthma as reference), allergy (no allergy as reference), BMI categorized as underweight/normal versus overweight/obese (under- weight/normal as reference), ever smoking (no smoking as reference), and current smoking (no smoking as reference). b Forest plot illustrating the association between glioma risk and potential confounding factors
IDH1 mutation and $1 \mathrm{p} / 19 \mathrm{q}$ co-deletion OR $=0.82 ; 95 \%$ CI 0.48-1.42) (Table 2). rLTL for all controls and cases, and for women and men respectively are illustrated in Supplementary Fig. 1.

\section{Telomere length and general health}

Subjects with allergies had a lower glioma risk $(\mathrm{OR}=0.61$; 95\% CI 0.45-0.83) (Fig. 1). We found no evidence that this association differed between high-grade and low-grade 
glioma cases (data not shown). When analyzing specific types of allergy symptoms, watery eyes $(\mathrm{OR}=0.48 ; 95 \%$ CI $0.28-0.82$ ) was significantly protective against glioma, whereas we found no statistically significant association between glioma risk and respiratory symptoms $(\mathrm{OR}=1.09 ; 95 \%$ CI $0.67-1.76)$ or eczema $(\mathrm{OR}=0.66 ; 95 \%$ CI 0.41-1.09) (Fig. 1). Asthma was not significantly associated with glioma risk ( $\mathrm{OR}=0.91 ; 95 \%$ CI 0.59-1.40) (Fig. 1, Supplementary Fig. 2). High BMI a year prior diagnosis was significantly protective against glioma $(\mathrm{OR}=0.67 ; 95 \% \mathrm{CI}$ $0.52-0.87$ ) (Fig. 1), and this association was not affected by the addition of rLTL to the model (data not shown). Smoking was not associated with glioma risk (Fig. 1). Adjusting for allergies, asthma, BMI, or smoking did not markedly change the association between rLTL and glioma risk (Fig. 1). Logistic regression models to investigate the interaction between rLTL and allergies, did not reveal any statistically significant effects on glioma risk $\left(P_{\text {interaction }}=0.948\right)$. rLTL for controls and cases, and the confounding factors are illustrated in Supplementary Figs. 2, 3 and 4. Among the controls in the Swedish cohort, we could not confirm previous studies showing shorter rLTL in individuals with allergic phenotypes (Supplementary Table 1).

\section{Telomere length and tumor characteristics}

Age at diagnosis and sex were the only factors associated with rLTL in linear regression analyses conducted to examine the association between rLTL and IDHI mutation, $1 \mathrm{p} / 19 \mathrm{q}$ co-deletion as well as EGFR amplification in the tumor (Table 3). An association between IDHI mutation and

\section{Discussion}

In this study, rLTL was measured in cases and controls, and the interplay between rLTL, asthma and allergies, and glioma molecular phenotype was investigated. Telomere length in the hematopoietic compartment was used as a proxy for average individual telomere length in non-malignant tissues, to explore individual susceptibility to disease as telomere length is determined by both genetic [29] and environmental factors $[30,31]$.

Our results reveal that longer rLTL is associated with increased risk of glioma. Although not always statistically significant, the association was in the same direction in all glioma subgroups, with the exception of glioma with both IDH1 mutation and 1p/19q co-deletion, suggesting that longer rLTL is not associated with risk of glioma with IDH1 mutation and $1 \mathrm{p} / 19 \mathrm{q}$ co-deletion (i.e., oligodendroglioma). Our finding of an association between longer rLTL and glioma risk confirms an earlier study investigating genotypically-estimated leukocyte telomere length (i.e., an estimation of telomere length based on a subject's genetic variants known to influence telomere length) and glioma risk [21]. Wang et al. [23] reported that both longer and shorter rLTL in peripheral blood are associated with higher risk of glioma, suggesting a U-shaped association between rLTL and glioma risk. On the other hand, Walcott et al. [22] reported no association overall, though found an association between short rLTL in peripheral blood and increased risk of glioma specifically among males. Contradictory to these reports, in this study we found a decreased risk of glioma in sub-
Table 3 Linear regression assessing associations between relative telomere length, age at diagnosis, sex, $I D H 1$ mutation and $1 \mathrm{p} / 19 \mathrm{q}$ co-deletion separately or in combination, and EGFR amplification for glioma cases. Each variable was considered separately (univariate analysis) and in a model combining all variables (multivariate analysis)

\begin{tabular}{|c|c|c|c|c|}
\hline & \multicolumn{2}{|l|}{ Univariate analysis } & \multicolumn{2}{|l|}{ Multivariate analysis } \\
\hline & Regression coefficient (SE) & $P$ value & Regression coefficient (SE) & $P$ value \\
\hline Age at diagnosis & $-0.004(0.001)$ & 0.0001 & $-0.003(0.001)$ & 0.0001 \\
\hline $\operatorname{Sex}^{\mathrm{a}}$ & $-0.046(0.020)$ & 0.021 & $-0.052(0.020)$ & 0.010 \\
\hline IDH1 mutation ${ }^{\mathrm{b}}$ & $0.064(0.027)$ & 0.017 & $0.023(0.039)$ & 0.558 \\
\hline $1 \mathrm{p} / 19 \mathrm{q}$ co-deletion ${ }^{\mathrm{c}}$ & $0.002(0.029)$ & 0.946 & $-0.027(0.040)$ & 0.507 \\
\hline $\begin{array}{l}\text { IDHI mutation and } \\
1 \mathrm{p} / 19 \mathrm{q} \text { co-deletion }\end{array}$ & $0.011(0.038)$ & 0.768 & $-0.019(0.064)$ & 0.771 \\
\hline$E G F R$ amplification ${ }^{\mathrm{e}}$ & $-0.039(0.021)$ & 0.066 & $-0.024(0.022)$ & 0.265 \\
\hline Adjusted $R$ square & & & 0.072 & \\
\hline$N$ & & & 298 & \\
\hline
\end{tabular}

${ }^{\mathrm{a}}$ Women used as reference category; ${ }^{\mathrm{b}} I D H 1$ mutation used as reference category; ${ }^{\mathrm{c}} 1 \mathrm{p} / 19 \mathrm{q}$ co-deletion used as reference category; ${ }^{\mathrm{d}} I D H 1$ mutation and $1 \mathrm{p} / 19 \mathrm{q}$ co-deletion used as reference category; ${ }^{\mathrm{e}} E G F R$ amplification used as reference category; All categories are coded as 0 versus 1
rLTL was noted in the univariate analysis, but this interaction disappeared when including age at diagnosis and sex in the model $(P=0.575)$. jects with short rLTL, specifically among females. Similarly, inconsistent results are not only restricted to glioma, since a number of studies have examined the relationship between telomere length measured in peripheral blood and various 
cancer risk with conflicting results. The majority of the studies show that short telomere length are associated with increased risk of cancer [32-36], while the opposite, with an association between long telomere length and increased cancer risk, has been shown by other studies [37-40]. The biological mechanisms behind the association between altered telomere length in peripheral blood and increased cancer risk is unclear. It is also not evident whether alterations in telomere length in peripheral blood are caused by the cancer disease, or if the alterations are actually part of the cancer etiology. It is evident that the appearance of a malignant disease affects the whole individual, and the alterations in telomere length in peripheral blood seen at diagnosis might reflect the responses to the tumor. One limitation of this study is that the glioma patients were recruited at, or even after, their diagnosis. Consequently, blood samples from the patients were drawn at a time when medical treatment, stress, and inflammatory conditions may have had an effect on rLTL in peripheral blood. But, since stress and inflammatory conditions are expected to shorten telomere length in patients [41], these confounding factors would not affect the association between longer telomere length and glioma risk. In addition, our findings are supported by the previously mentioned study by Walsh et al. [21] in which telomere length was estimated based on genotypes (i.e., a Mendelian randomization approach), which would be independent of confounding factors such as medical treatment.

In concordance with previous studies, we report that individuals with allergies have a decreased risk of glioma [7, 42, 43] and allergy symptoms like watery eyes appeared to be more protective. In another GICC study by Amirian et al. 2016, where all the Swedish glioma cases- and controls were included, they reported that asthma and eczema were protective against glioma and that the association was significantly stronger in highgrade compared to low-grade glioma. Our data do not support the difference observed between high- and lowgrade glioma, which might be explained by the fact that we analyzed a slightly smaller number of glioma cases- and controls from the Swedish cohort. In addition, although the association between glioma risk and eczema was not statistically significant in the present study, it was in the expected direction [7]. One hypothesis why allergy seems to be protective against glioma is that the reactive state of the immune system in atopic individuals may result in increased immune surveillance and limit abnormal cell proliferation, though the specific mechanism by how this could limit abnormal cell proliferation and thereby tumor growth remains to be clarified [44].

Shorter rLTL in peripheral blood has recently been proposed to be strongly associated with metabolic syndrome, especially in the female population [45]. Likewise, in a longitudinal study reported by Lee et al. [46], rLTL in peripheral blood was shorter in an obese population. Our results propose that patients with a higher BMI one year prior diagnosis have decreased risk of glioma, whereas recently published data show that overweight or obesity are not connected to glioma risk [47]. The fact that this association was stronger for a high BMI one year prior to diagnosis, compared to the same association for BMI as adolescent or adult, raise the concern for reverse causality, as a low BMI may be coupled to undiagnosed disease. Although smoking has been suggested as a risk factor for glioma in some studies [48, 49], most studies conducted so far, including the present study [50-52], do not support an association between smoking and glioma risk.

IDHI mutation, frequently co-occurring with molecular alterations including $1 \mathrm{p} / 19 \mathrm{q}$ co-deletion, is an early event in the development of glial tumors and often accompanied by mutations influencing telomerase activity [53]. The tight linkage between IDHI mutations and mutations of genes and promoters involved in telomere regulation suggests that IDHI mutation may contribute to the genesis or selection of events that resolve telomeric dysfunction in the tumor, which may affect both immortalization and transformation [53]. Though, in this study, we did not find any association between rLTL measured in peripheral blood and IDHI mutation or other molecular alterations in the tumor.

A limitation with our study is that telomere length was measured in leukocytes and not in brain tissue. Although several studies have shown intra-individual synchrony in telomere length across somatic tissues in fast (e.g., blood and skin) as well as slowly proliferating tissues (e.g., fat, muscle, synovium), there is a lack of robust studies which have correlated leukocyte telomere length with normal and malignant brain tissue $[54,55]$. Average rLTL may be influenced by immune response mediated telomerase activation, and TERT promoter mutations may influence telomere length in glioma tissue. Therefore, future studies measuring telomere length in glioma tissue will contribute to further understanding of the role of telomere biology in glioma pathology [56].

In conclusion, our study showed an association between long rLTL and risk of glioma. Even if previous studies have shown that asthma, BMI and smoking have an impact on telomere length, we were not able to establish any interactions of these risk factors in our cohort. Our study of measured rLTL support recent studies that associates high genetically estimated telomere length scores with increased glioma risk $[57,58]$. This further indicates that telomere biology is involved in the complex pathways of gliomagenesis.

Acknowledgments The tumor tissue slides are recruited from the different GICC study centers in Sweden, and Sara Huggert, Gudrun Byström, Eva Svedberg and Sonia Katsuura at the Oncology Clinic, Umea University Hospital, Umeå, Sweden are acknowledged for their assistance in this part of the study. We acknowledge Georgina N. Armstrong at the department of Medicine, Dan L. Duncan Comprehensive 
Cancer Center, Baylor College of Medicine, Houston, Texas, USA, for her valuable support in this study. We want to thank professor Thomas Brännström at the department of Medical Biosciences, Umea University, Umeå, Sweden for adequately reviewing all the tumor tissue slides. For laboratory technical assistance we want to thank Susann Haraldsson at the department of Medical Biosciences, Umeå University, for the telomere length measurements. Jonas Sörlin, Charlotte Andersson and Angelika Brännlund at Clinical Genetics, Umeå University Hospital, for the FISH analysis. Ulla-Stina Spetz at the department of Medical Bioscience, Umeå University, for the IHC analyses. Mikael Kimdal at the department of Radiation Sciences, Umeå University, for the extraction of DNA from blood.

Funding This study was funded by the National Cancer Institute NIH, (Grants/Award Number: 5R01 CA119215, 5R01CA139020). For more information about the Gliogene Consortium, refer to the following Web site: http://www.gliogene.org. The analyses were supported by the Swedish Cancer Foundation, Swedish Research Council, Umeå Hospital Cutting Edge Grant, The Northern Sweden Cancer foundation and Umeå University Hospital Grant. We also thank the patients and their families for participating in this study.

Open Access This article is distributed under the terms of the Creative Commons Attribution 4.0 International License (http://creativeco mmons.org/licenses/by/4.0/), which permits unrestricted use, distribution, and reproduction in any medium, provided you give appropriate credit to the original author(s) and the source, provide a link to the Creative Commons license, and indicate if changes were made.

\section{References}

1. Johansson G, Andersson U, Melin B (2016) Recent developments in brain tumor predisposing syndromes. Acta Oncol 55(4):401-411

2. Bondy ML et al (2008) Brain tumor epidemiology: consensus from the brain tumor epidemiology consortium. Cancer 113(7 Suppl):1953-1968

3. Ostrom QT et al (2014) The epidemiology of glioma in adults: a "state of the science" review. Neuro Oncol 16(7):896-913

4. Schlehofer B et al (1999) Role of medical history in brain tumour development. Results from the international adult brain tumour study. Int J Cancer 82(2):155-160

5. Wigertz A et al (2007) Allergic conditions and brain tumor risk. Am J Epidemiol 166(8):941-950

6. Schlehofer B et al (2011) Primary brain tumours and specific serum immunoglobulin E: a case-control study nested in the European Prospective Investigation into cancer and nutrition cohort. Allergy 66(11):1434-1441

7. Amirian ES et al (2016) Approaching a Scientific Consensus on the Association between Allergies and Glioma Risk: A Report from the Glioma International Case-Control Study. Cancer Epidemiol Biomarkers Prev 25(2):282-290

8. Shete $\mathrm{S}$ et al (2009) Genome-wide association study identifies five susceptibility loci for glioma. Nat Genet 41(8):899-904

9. Andersson U et al (2010) A comprehensive study of the association between the EGFR and ERBB2 genes and glioma risk. Acta Oncol 49(6):767-775

10. Rajaraman P et al (2012) Genome-wide association study of glioma and meta-analysis. Hum Genet 131(12):1877-1888

11. Walsh KM et al (2014) Variants near TERT and TERC influencing telomere length are associated with high-grade glioma risk. Nat Genet 46(7):731-735
12. Bainbridge $\mathrm{MN}$ et al (2015) Germline mutations in shelterin complex genes are associated with familial glioma. J Natl Cancer Inst 107(1):384

13. Melin BS et al (2017) Genome-wide association study of glioma subtypes identifies specific differences in genetic susceptibility to glioblastoma and non-glioblastoma tumors. Nat Genet 49(5):789

14. Belsky DW et al (2014) Is chronic asthma associated with shorter leukocyte telomere length at midlife? Am J Respir Crit Care Med 190(4):384-391

15. Valdes AM et al (2005) Obesity, cigarette smoking, and telomere length in women. Lancet 366(9486):662-664

16. Blackburn EH, Epel ES, Lin J (2015) Human telomere biology: a contributory and interactive factor in aging, disease risks, and protection. Science 350(6265):1193-1198

17. McDonald KL et al (2010) Presence of alternative lengthening of telomeres mechanism in patients with glioblastoma identifies a less aggressive tumor type with longer survival. J Neuropathol Exp Neurol 69(7):729-736

18. Louis DN et al (2016) The 2016 World Health Organization Classification of Tumors of the Central Nervous System: a summary. Acta Neuropathol 131(6):803-820

19. Lotsch $D$ et al (2013) Prognostic significance of telomerase-associated parameters in glioblastoma: effect of patient age. Neuro Oncol 15(4):423-432

20. Heeg $\mathrm{S}$ et al (2011) EGFR overexpression induces activation of telomerase via PI3K/AKT-mediated phosphorylation and transcriptional regulation through Hif1-alpha in a cellular model of oral-esophageal carcinogenesis. Cancer Sci 102(2):351-360

21. Walsh KM et al (2015) Longer genotypically-estimated leukocyte telomere length is associated with increased adult glioma risk. Oncotarget 6(40):42468-42477

22. Walcott $\mathrm{F}$ et al (2013) Telomere length and risk of glioma. Cancer Epidemiol 37(6):935-938

23. Wang $\mathrm{S}$ et al (2014) Association between leukocyte telomere length and glioma risk: a case-control study. Neuro Oncol 16(4):505-512

24. Walsh KM et al (2015) Telomere maintenance and the etiology of adult glioma. Neuro Oncol 17(11):1445-1452

25. Malmer B et al (2007) GLIOGENE an International Consortium to Understand Familial Glioma. Cancer Epidemiol Biomarkers Prev 16(9): 1730-1734

26. Amirian ES et al (2016) The glioma international case-control study: a report from the Genetic Epidemiology of Glioma International Consortium. Am J Epidemiol 183(2):85-91

27. Cawthon RM (2009) Telomere length measurement by a novel monochrome multiplex quantitative PCR method. Nucleic Acids Res 37(3):e21

28. Ghasimi $S$ et al (2016) Genetic risk variants in the CDKN2A/B, RTEL1 and EGFR genes are associated with somatic biomarkers in glioma. J Neurooncol 127(3):483-492

29. Bekaert S, De Meyer T, Van Oostveldt P (2005) Telomere attrition as ageing biomarker. Anticancer Res 25(4):3011-3021

30. Epel ES et al (2004) Accelerated telomere shortening in response to life stress. Proc Natl Acad Sci USA 101(49):17312-17315

31. von Zglinicki T (2002) Oxidative stress shortens telomeres. Trends Biochem Sci 27(7):339-344

32. Jang JS et al (2008) Telomere length and the risk of lung cancer. Cancer Sci 99(7):1385-1389

33. Shen J et al (2007) Short telomere length and breast cancer risk: a study in sister sets. Cancer Res 67(11):5538-5544

34. Mirabello L et al (2011) Telomere length and variation in telomere biology genes in individuals with osteosarcoma. Int J Mol Epidemiol Genet 2(1):19-29

35. Widmann TA et al (2007) Short telomeres in aggressive nonHodgkin's lymphoma as a risk factor in lymphomagenesis. Exp Hematol 35(6):939-946 
36. $\mathrm{Ma} \mathrm{H}$ et al (2011) Shortened telomere length is associated with increased risk of cancer: a meta-analysis. PLoS One 6(6):e20466

37. Han J et al (2009) A prospective study of telomere length and the risk of skin cancer. J Invest Dermatol 129(2):415-421

38. Svenson U, Ljungberg B, Roos G (2009) Telomere length in peripheral blood predicts survival in clear cell renal cell carcinoma. Cancer Res 69(7):2896-2901

39. Gramatges MM et al (2010) Longer relative telomere length in blood from women with sporadic and familial breast cancer compared with healthy controls. Cancer Epidemiol Biomarkers Prev 19(2):605-613

40. Lan Q et al (2013) Longer telomere length in peripheral white blood cells is associated with risk of lung cancer and the rs2736100 (CLPTM1L-TERT) polymorphism in a prospective cohort study among women in China. PLoS ONE 8(3):e59230

41. Jose SS et al (2017) Chronic inflammation in immune aging: role of pattern recognition receptor crosstalk with the telomere complex? Front Immunol 8:1078

42. Chen $\mathrm{C}$ et al (2011) Allergy and risk of glioma: a meta-analysis. Eur J Neurol 18(3):387-395

43. Zhao $\mathrm{H}$ et al (2014) Allergic conditions reduce the risk of glioma: a meta-analysis based on 128,936 subjects. Tumour Biol 35(4):3875-3880

44. Linos E et al (2007) Atopy and risk of brain tumors: a metaanalysis. J Natl Cancer Inst 99(20):1544-1550

45. Cheng YY et al (2017) Examining the gender difference in the association between metabolic syndrome and the mean leukocyte telomere length. PLoS ONE 12(7):e0180687

46. Lee $\mathrm{M}$ et al (2011) Inverse association between adiposity and telomere length: the Fels longitudinal study. Am J Hum Biol 23(1):100-106

47. Wiedmann MKH et al (2017) The impact of body mass index and height on the risk for glioblastoma and other glioma subgroups: a large prospective cohort study. Neuro Oncol 19(7):976-985
48. Efird JT et al (2004) The risk for malignant primary adult-onset glioma in a large, multiethnic, managed-care cohort: cigarette smoking and other lifestyle behaviors. J Neurooncol 68(1):57-69

49. Silvera SA, Miller AB, Rohan TE (2006) Cigarette smoking and risk of glioma: a prospective cohort study. Int $\mathrm{J}$ Cancer 118(7): $1848-1851$

50. Holick CN et al (2007) Prospective study of cigarette smoking and adult glioma: dosage, duration, and latency. Neuro Oncol 9(3):326-334

51. Lachance DH et al (2011) Associations of high-grade glioma with glioma risk alleles and histories of allergy and smoking. Am J Epidemiol 174(5):574-581

52. Braganza MZ et al (2014) Cigarette smoking, alcohol intake, and risk of glioma in the NIH-AARP Diet and Health Study. Br J Cancer 110(1):242-248

53. Ohba $\mathrm{S}$ et al (2016) Mutant IDH1 expression drives TERT promoter reactivation as part of the cellular transformation process. Cancer Res 76(22):6680-6689

54. Friedrich $\mathrm{U}$ et al (2000) Telomere length in different tissues of elderly patients. Mech Ageing Dev 119(3):89-99

55. Daniali L et al (2013) Telomeres shorten at equivalent rates in somatic tissues of adults. Nat Commun 4:1597

56. Huang DS et al (2015) Recurrent TERT promoter mutations identified in a large-scale study of multiple tumour types are associated with increased TERT expression and telomerase activation. Eur J Cancer 51(8):969-976

57. Randomization TM (2017) C., et al., Association between telomere length and risk of cancer and non-neoplastic diseases: a Mendelian randomization study. JAMA Oncol 3(5):636-651

58. Codd V et al (2013) Identification of seven loci affecting mean telomere length and their association with disease. Nat Genet 45(4):422-427, 427e1-2 